Elisabetta Versace ${ }^{1 *}$, Anna Eriksson ${ }^{1}$, Federico Rocchi ${ }^{1}$, Irene Castellan ${ }^{1,2,3}$, Paola Sgadò ${ }^{1}$, Albrecht Haase ${ }^{1,4}$

${ }^{1}$ Center for Mind/Brain Sciences, University of Trento

2 Department of Agricultural Sciences, University of Bologna

${ }^{3}$ Faculty of Science and Technology, Free University of Bozen/Bolzano

${ }^{4}$ Department of Physics, University of Trento

*Corresponding author: elisabetta.versace@unitn.it

\title{
Physiological and behavioral responses in Drosophila melanogaster to odorants present at different plant maturation stages.
}

\begin{abstract}
The fruit fly Drosophila melanogaster feeds and oviposits on fermented fruit, hence its physiological and behavioral responses are expected to be tuned to odorants abundant during later stages of fruit maturation. We used a population of about two-hundred isogenic lines of D. melanogaster to assay physiological responses (electroantennograms (EAG)) and behavioral correlates (preferences and choice ratio) to odorants found at different stages of fruit maturation. We quantified electrophysiological and behavioral responses of $D$. melanogaster for the leaf compound $\beta$-cyclocitral, as well as responses to odorants mainly associated with later fruit maturation stages. Electrophysiological and behavioral responses were modulated by the odorant dose. For the leaf compound we observed a steep doseresponse curve in both EAG and behavioral data and shallower curves for odorants associated with later stages of maturation. Our data show the connection between sensory and behavioral responses and are consistent with the specialization of D. melanogaster on fermented fruit and avoidance of high doses of compounds associated with earlier stages of maturation. Odor preferences were modulated in a non-additive way when flies were presented with two alternative odorants, and combinations of odorants elicited higher responses than single compounds.
\end{abstract}

\section{Keywords}

Olfaction, Drosophila melanogaster, electrophysiology, electroantennography, T-maze, behavioral preferences, attraction, aversion, unlearned preferences, fruit maturation, Drosophila Genetic Reference Panel (DGRP), $\beta$-cyclocitral, isoamyl acetate, ethyl 3hydroxybutyrate 


\section{INTRODUCTION}

Olfactory cues are a powerful drive for feeding, mating, and oviposition in fruit flies [1-5]. Specialization at the level of olfactory receptors and behavioral responses has been well documented in Drosophila [2,3]: for instance, different drosophilids' preference for yeast which supports best their growth and survival [6,7], and genomic adaptation [8], or olfactory genes sustaining the attraction of Drosophila sechellia by the odor of its obligate fruit host [9], which is repellent and lethal for other species [10]. Beside being used to investigate the genetic basis of olfactory behavior [11,12], Drosophila melanogaster has recently gained greater relevance due to the threat on agriculture posed by its cousin species Drosophila suzukii [13-15]. This pest is invading western countries from Asia [16] and its habitat partially overlaps with $D$. melanogaster. While both species feed on fermented media, only $D$. suzukii oviposits on ripe small fruits. Thus, investigating sensory and behavioral responses in Drosophila for compounds corresponding to different stages of fruit maturation is of primary interest for both basic and applied research. Keesey et al. [17] recently tested whether the ecological shift of $D$. suzukii, but not $D$. melanogaster, towards ripe fruit is influenced by sensory tuning of this species to odorants present in leaves, such as the isoprenoid volatile $\beta$ cyclocitral. They found that only $D$. suzukii shows electrophysiologic responses to $\beta$-cyclocitral and is lured by traps containing this compound. On this basis, Keesey et al. [17] argued that the specialization of the invasive species $D$. suzukii for ripe fruit is sustained by a speciesspecific sensitivity to leaf compounds, which are associated with early stages of fruit maturation. To better understand the role of odorant type and dose in eliciting sensory and behavioral responses, in our assays we tested $D$. melanogaster using the same leaf compound $\beta$-cyclocitral and odorants associated with different stages of fruit maturation [17-20], presented in increasing amounts.

As ripening fruit compounds we used isoamyl acetate, which is consistently present in ripening fruit and active in both D. melanogaster and D. suzukii [18], and other volatiles present during fruit maturation: ethyl butyrate (EB, > 97\% purity) [19,21], and 2-methylbutyl-acetate (MBA, > 99\% purity) [19,22]. Among the fermented fruit compounds we used methyl and ethyl hexanoate, which are known as the best ligands for the receptor Or22a in $D$. melanogaster (whereas they elicit a reduced response in D. suzukii), and ethyl-3hydroxybutyrate, which elicits electrophysiological responses on the ab2 sensillum in the Drosophila melanogaster subgroup [23]. Single compounds do not exactly match the complex fruit headspace [e.g. 24,25,26] but can be used as "placeholders" that allow standardized manipulation of compound doses and replicability.

We first measured electroantennographic responses, showing that $D$. melanogaster indeed responds to the green leaf compound $\beta$-cyclocitral [17], in addition to the other presented compounds. Using a static T-maze [27], we tested groups of 100 individuals (males and females) with different haplotypes, in order to have a representation of the entire population (see [28] for an investigation of the dynamics of collective decisions in groups of 4 to 200 flies). Although this setting does not distinguish between the contribution of males and females, it provides a representative overview of flies' behavior in the presence of group interactions. This is particularly important for a species that frequently aggregates on food. We assessed the attraction of the most effective compound of each fruit maturation stage presented in different amounts, compared to a blank control, showing which dose of each odorant is attractive or aversive. We also measured the proportion of flies lured out from the starting point into the T-maze (choice ratio). Subsequently, we compared the relative attraction between pairs of odorants and the proportion of flies lured out from the starting point when two alternative odorants were presented and when two odorants were presented simultaneously against a blank control. 


\section{MATERIALS AND METHODS}

\section{Drosophila stocks}

We used adult fruit flies from 195 lines of the Drosophila Genetic Reference Panel (DGRP) [29]. This population consists of isofemale lines generated by 20 generations of full-sib mating of lines started from inseminated females of the wild-caught Raleigh (North Carolina) population described by Mackay et al. [29]. Arya et al. [11] have recently documented the genetic basis of olfactory variability in this fully sequenced population.

\section{Electroantennography}

In this assay, data from 28 antennae of 2-7 days old individuals of different lines (15 males and 13 females) were recorded. We measured electroantennograms (EAGs) through a standard apparatus (Syntech, Hilversum, NL) connected to the antenna through microcapillary glass electrodes filled with Kaissling saline solution containing $5 \mathrm{~g} / \mathrm{l}$ polyvinylpyrrolidone. The head of the insect was mounted on a microcapillar connected to a ground silver electrode, while the recording silver electrode was placed at the tip of one antenna. Each compound was delivered to the antenna through a glass tube $(12 \mathrm{~cm} \times 8 \mathrm{~mm})$ via a constant humidified air stream $(0.5 \mathrm{l} / \mathrm{m})$ filtered with charcoal. The air tube was located 4-5 mm away from the antenna. The test cartridge was connected to a stimulus controller (CS55 , Syntech) that generated the air puffs. As odorants we used synthetic compounds (purchased from Sigma Aldrich, Italy, with maximum purity available) that in nature are more abundant at subsequent maturation stages of a plant (see Table 1) $[17,18]$ : one leaf odorant for early fruit maturation: $\beta$-cyclocitral (BCC, > 95\% purity) [17]; three odorants typical of ripeninig and fermented fruit stage: isoamyl acetate (IAA, $>99 \%$ purity) $[18,19,30]$, ethyl butyrate (EB, > 97\% purity) [19,21], 2-methyl-butyl-acetate (MBA, > 99\% purity) [19,22]; and three odors strongly associated with fermented fruit: ethyl 3-hydroxybutyrate (E3HB, > 97\% purity) [19], methyl hexanoate ( $\mathrm{MH},>99 \%$ purity) [17], ethyl hexanoate ( $\mathrm{EH},>99 \%$ purity) $[17,31-33]$. Stimuli were diluted in hexane to four different concentrations $\left(10^{-4}, 10^{-3}, 10^{-2}\right.$, $10^{-1} \mu \mathrm{g} / \mu \mathrm{l}$ ), and $10 \mu \mathrm{l}$ were put on $1.5 \mathrm{~cm}^{2}$ piece of filter paper (Albet 400: Sparks Lab Supply Limited) inside Pasteur pipettes, after the solvent was let to evaporate for a couple of minutes. Cartridges were replaced after about ten puffs. To avoid biases in the response amplitude we varied the order of the odorants during the presentation among individuals. For each individual we delivered odorants in ascending order of concentration at 30-40 s intervals. Each odorant and concentration was presented only once to each individual. As control stimuli we used pure hexane and blank air at the beginning, the middle, and the end of each recording. Electroantennographic responses were analyzed with the EAG2000 software (Syntech), by measuring the maximum amplitude of depolarization [mV] of each stimulus. Data were log-transformed to obtain a normal distribution. 


\begin{tabular}{|l|l|l|l|}
\hline Maturation stage & Compound & Acronym & $\begin{array}{l}\text { Vapor pressure } \\
{[\mathrm{mmHg}]}\end{array}$ \\
\hline Green leaf & -cyclocitral & BCC & 0.032 \\
\hline $\begin{array}{l}\text { Ripe fruit } \\
\text { (ripening/fermenting) }\end{array}$ & isoamyl acetate & IAA & 5.60 \\
\hline $\begin{array}{l}\text { Ripe fruit } \\
\text { (ripening/fermenting) }\end{array}$ & ethyl butyrate & EB & 12.8 \\
\hline $\begin{array}{l}\text { Ripe fruit } \\
\text { (ripening/fermenting) }\end{array}$ & 2-methyl-butyl-acetate & MBA & 7.85 \\
\hline $\begin{array}{l}\text { Fermented fruit } \\
\text { (fermenting/ripening) }\end{array}$ & ethyl 3-hydroxybutyrate & E3HB & 0.36 \\
\hline $\begin{array}{l}\text { Fermented fruit } \\
\text { (fermenting/ripening) }\end{array}$ & methyl hexanoate & MH & 3.95 \\
\hline $\begin{array}{l}\text { Fermented fruit } \\
\text { (fermenting/ripening) }\end{array}$ & ethyl hexanoate & EH & 1.66 \\
\hline
\end{tabular}

Table 1.

Stage of fruit maturation for each odorant used, its acronym and vapor pressure.

\section{T-maze assays}

\section{Subjects}

In each trial and T-maze we tested a group of 100 adult flies of both sexes collected in groups of 12-15 individuals from different isofemale lines of our stock (see Drosophila stocks).

\section{Apparatus and experimental procedure}

The glass T-maze $(14 \times 10 \mathrm{~cm}, \varnothing 8 \mathrm{~mm}$, junctions' $\varnothing 2 \mathrm{~mm})$ used for the experimental assays (Figure 1) was connected to a starting chamber (a standard Drosophila vial, $9 \times 2.5 \mathrm{~cm}$ ) and to two odor chambers located on each side of the T-maze. The apparatus was placed horizontally. Odor chambers (standard drosophila vials) contained $20 \mu \mathrm{l}$ of the test compounds pipetted on $1 \times 2 \mathrm{~cm}$ pieces of filter paper. The test compound was obtained through consecutive dilutions of the pure odorant in distilled water (water as solvent to dilute odorants for behavioral experiments had been previously used in a large study with hundreds of odorants in D. melanogaster [2] and is easy to handle in large-scale studies). To make sure to present the desired dose for each compound irrespectively of its water solubility, we vortexed each solution before pipetting it on the filter paper. Each odor chamber contained a different odorant that diffused into the apparatus without air flow from outside. Efficient diffusion of odorants into the central chamber, was shown in similar experiments investigating olfactory learning and preferences in fruit flies [27] and is confirmed by our results. Despite the lack of controllable airflow, a sufficient diffusion of the compounds through the apparatus is confirmed by the dose-response curves showing that flies could detect changes in dosage. This confirms that this setup is particularly suitable for high throughput tests [27]. 


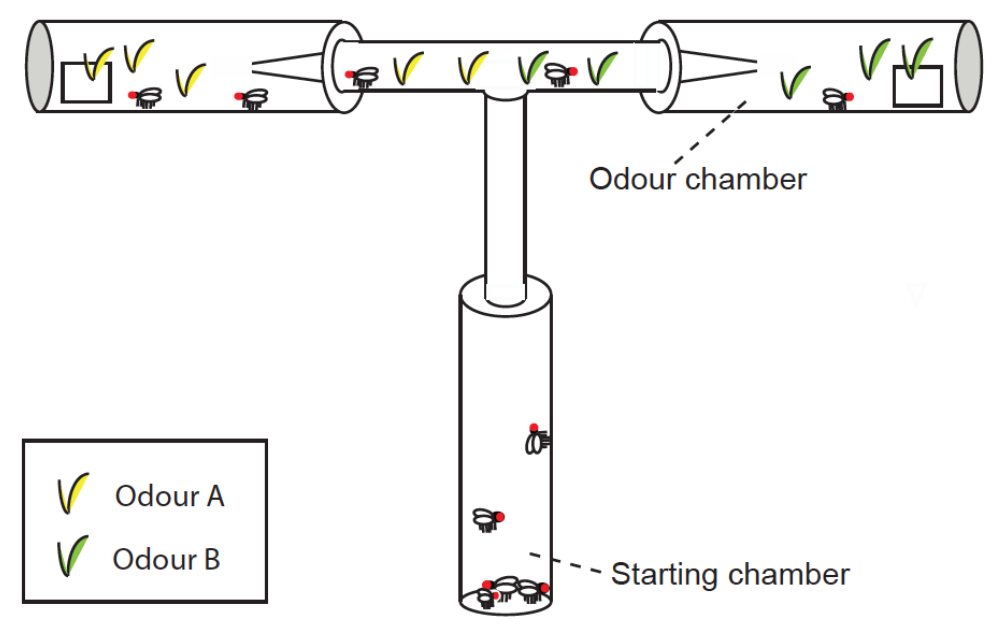

Figure 1.

T-maze used for behavioral assays (which is oriented with all chambers in the horizontal plane). At the beginning of the test, a group of 100 flies is located in the starting chamber. Flies are free to explore the apparatus and to enter the lateral arms (odor chambers) of the T-maze. Odor chambers contain the odorants that diffuse through the apparatus.

After 4 hours of food deprivation in a standard drosophila vial, the flies were released into the apparatus and could explore the T-maze for 60 minutes before the experiment was terminated. After half of the time, we removed and substituted the odor chambers with new vials containing the same odorant, to prevent flies from making a second choice.

During the trial, each T-maze was located in an isolated chamber $(50 \times 40 \times 20 \mathrm{~cm})$ with light symmetrically illuminating the apparatus. At the end of the trial, we counted the number of flies that entered each odor chamber in the first and second period and the number of alive flies that did not leave the starting vial. With these data we calculated:

- Olfactory preference $=($ number of flies that chose odorant $A) /($ number of flies that chose the odorant A + number of flies that chose odorant B (or blank))

- Choice ratio $=$ (number of flies that entered one of the odor chambers $) /($ number of flies that entered one of the odor chambers + number of alive flies that did not enter any odor chamber).

\section{Odor vs. Blank experiment}

In the first experiment, we tested the preference for an odorant (BCC, IAA, or E3HB) vs. Blank and the choice ratio (ratio of flies that entered the odor chambers) in response to five odor doses: $0.002,0.02,0.2,1,2 \mu \mathrm{g}$. We also ran a control experiment of 36 replicates to establish the baseline for the choice rate in the absence of odorants, measuring the attraction for flies exposed to two blank vials (Blank vs. Blank). For each odorant and dose we ran a minimum of 20 independent trials (see Supplementary table 1).

\section{Odor vs. Odor experiment}

In the second experiment, we tested the relative preference for odorants presented in pairs of the same dose $(0.2,1,2 \mu \mathrm{g})$ in different arms of the T-maze and the overall attraction. We used a representative odorant of each maturation stage (see Table 1): $\beta$-cyclocitral (BCC) for leaf, isoamyl acetate (IAA) for ripening fruit, and ethyl 3-hydroxybutyrate (E3HB) for overripe/fermenting fruit. For each odor-pair and dose we ran a minimum of 20 independent trials (see Supplementary table 2).Same odors experiment 
In this experiment we tested the choice ratio elicited in the presence of two identical odorants (BCC, IAA or E3HB) presented with the same dose $(0.002,0.02,0.2,1,2 \mu \mathrm{g})$ in both odor chambers of the T-maze. For each odor and dose we ran 20 independent trials (see Supplementary table 3)

\section{Two odors vs. Blank experiment}

In this experiment we tested the olfactory preference and choice elicited by a pair of odorants (BCC and IAA, E3HB and BCC, E3HB and IAA) presented in the same arm vs. Blank. Each odorant was presented at same dose $0.2,1$ or $2 \mu \mathrm{g}$. For each odor-pair and dose we ran at least 20 independent trials (see Supplementary table 4).

\section{Data analysis}

For the electroantennographic recordings, after normalization of the distribution of data (amplitude of depolarization in $\mathrm{mV}$ ) using a log-transformation, we used ANOVA with Dose, Stimulus, and Maturation stage as within factors, and Sex as between factor. Alpha level was set to 0.05 . We used Welch-corrected two sample $t$-tests to assay differences between specific odours and doses.

For the T-maze assays, non-normal distributed data were normalized using an arcsintransformation. We analysed the data (olfactory preference, choice ratio) using ANOVA with Stimulus and Dose as between factors. Alpha level was set to 0.05. Preferences for one compound were tested using one-sample $t$-tests against the chance level, which was 0.5 for the odour preferences and 0.71 for the choice ratio (this value was established testing fruit flies with two control stimuli that contained only water).

\section{RESULTS}

\section{Electroantennography}

After log-transformation, data approached the normal distribution (we removed 6 outliers, outside 1.5 times the interquartile range above the upper quartile or below the lower quartile). The analysis of variance showed a significant main effect of Stimulus $\left(F_{6,151}=17.04, p<0.001\right)$ and Concentration $\left(F_{3,540}=50.1, p<0.001\right)$ and no effect of $\operatorname{Sex}\left(F_{1,23}=0.01, p=0.98\right)$. We observed only one significant interaction: Stimulus $\times$ Concentration $\left(F_{18,540}=3.44, p<0.001\right)$, see Figure 2.

To check whether each compound was detected, eliciting higher responses than the control hexane, we tested odor groups against this control using Welch-corrected two-sample $t$-tests. Considering all concentrations, all stimuli - including the leaf compound BCC - showed significantly higher responses compared to control (the response to blank control was lower with respect to hexane). We obtained the lowest response from BCC at concentration $10^{-4}$. While at this concentration the recordings had only a trend towards higher responses for BCC compared to hexane $(t=1.59, d f=63.1, p=0.12)$, at concentration $10^{-3}$ flies clearly responded stronger to BCC than to control $(t=3.74, d f=52.7, p<0.001)$. All other compounds elicited significantly different responses with respect to control starting from the lowest concentration.

To investigate differences between the subsequent stages of Maturation (leaf, ripening fruit, fermented fruit) and concentrations we performed a second ANOVA. Data showed a significant difference between stages of Maturation $\left(F_{2,50}=15.9, p<0.001\right)$ and a significant interaction Maturation $\times$ Concentration $\left(F_{6,162}=6.79, p<0.001\right)$. The dependence on the Maturation factor shows an increase in amplitude response at later maturation stages. We 
checked this pattern with Welch-corrected two-sample $t$-tests: overall, the difference between leaf and ripe fruit stage trends to higher responses for later maturation stage $(t=-1.29, d f=$ $192, p=0.20$ ), and the ripening stage elicits significantly lower responses than the overripe/fermented stage $(t=-3.75, d f=664, p<0.001)$.

Response amplitude by Stimulus and Concentration

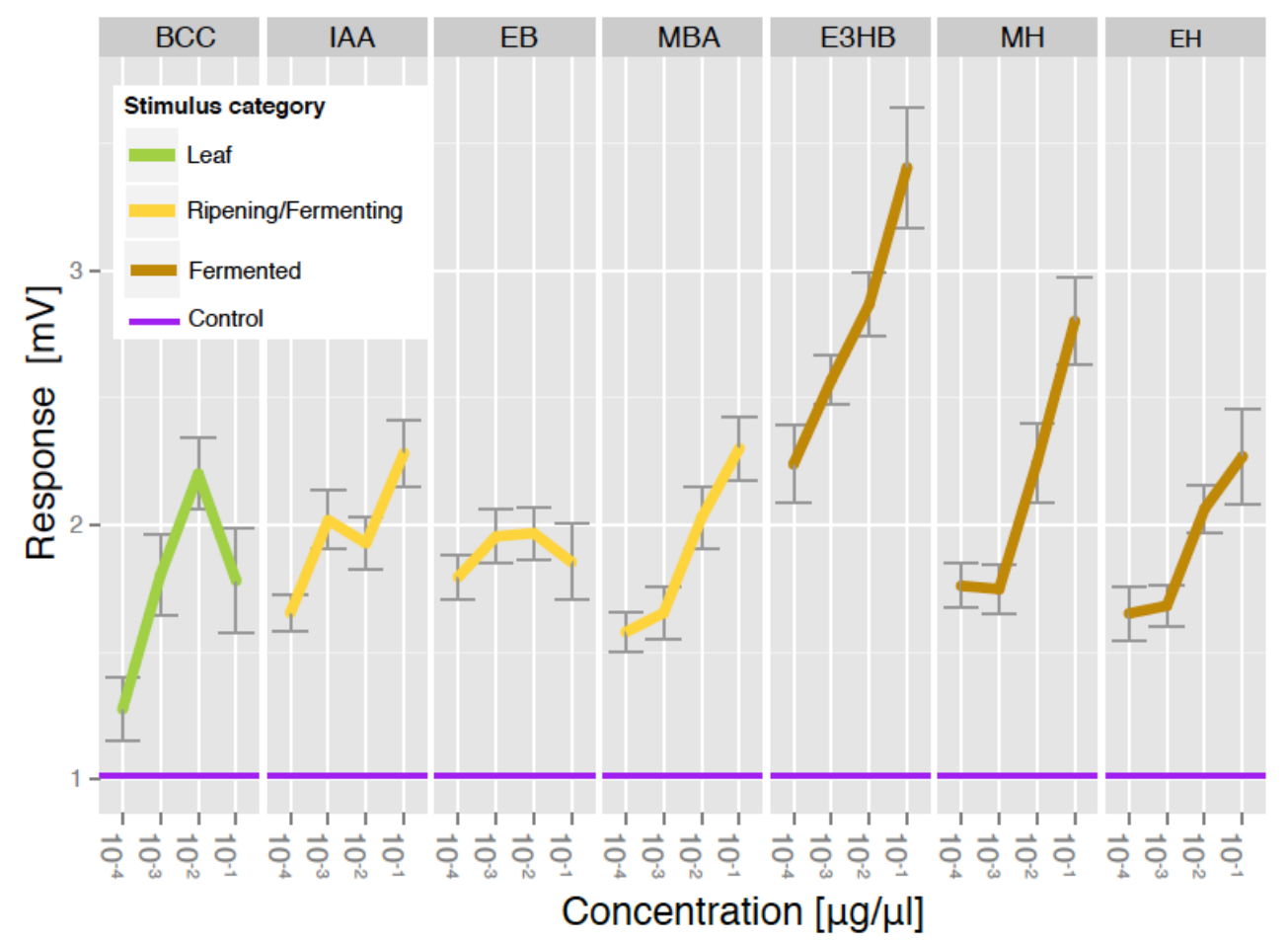

Figure 2.

EAG absolute responses (Mean \pm SEM in $\mathrm{mV}$ ) to different compounds as a function of Stimulus and Concentration. Compounds tested are indicated on top of each plot: $\mathrm{BCC}=\beta$-cyclocitral, IAA = isoamyl acetate, $\mathrm{EB}=$ ethyl butyrate, $\mathrm{MBA}=$ 2-methyl-butyl-acetate, $\mathrm{E} 3 \mathrm{HB}=$ ethyl 3-hydroxybutyrate, $\mathrm{MH}=$ methyl hexanoate, $\mathrm{EH}$ = ethyl hexanoate. The purple line shows the baseline response for the control stimulus (pure hexane).

\section{T-maze behavioral assays: Odor vs. Blank experiment}

To analyze the olfactory preference for the odorant $v s$. blank we used the ANOVA, testing for effects of Stimulus, Dose, and their interaction. Exploratory data analyses revealed three outliers (values outside 1.5 times the interquartile range above the upper quartile or below the lower quartile), that we removed for the final analyses. In the preference tests for odor vs. blank we observed a significant main effect of Stimulus $\left(F_{2,387}=8.718, p<0.001\right)$ and Dose $\left(F_{4,387}=7.746, p<0.001\right)$ and a significant interaction Stimulus $\times$ Dose $\left(F_{8,387}=2.45, p=0.013\right)$, see Figure 3.

For each compound, we used one-sample $t$-tests with Bonferroni-Holm correction (from $\alpha<0.05$ ) to assess whether flies exhibited attraction or aversion towards different odorant doses (see Table 2), starting from dose $0.02 \mu \mathrm{g}$. For BCC, a dose of $1 \mu \mathrm{g}$ was attractive while a dose of $2 \mu \mathrm{g}$ was strongly aversive. Hence, although the overall performance with BCC was not significantly different from chance $\left(t_{128}=-0.39, p=0.70\right.$, Mean $=0.50$, SEM $\left.=0.009\right)$, flies behaved in a qualitatively different way (attraction or aversion) in response to specific doses of the same compound. 


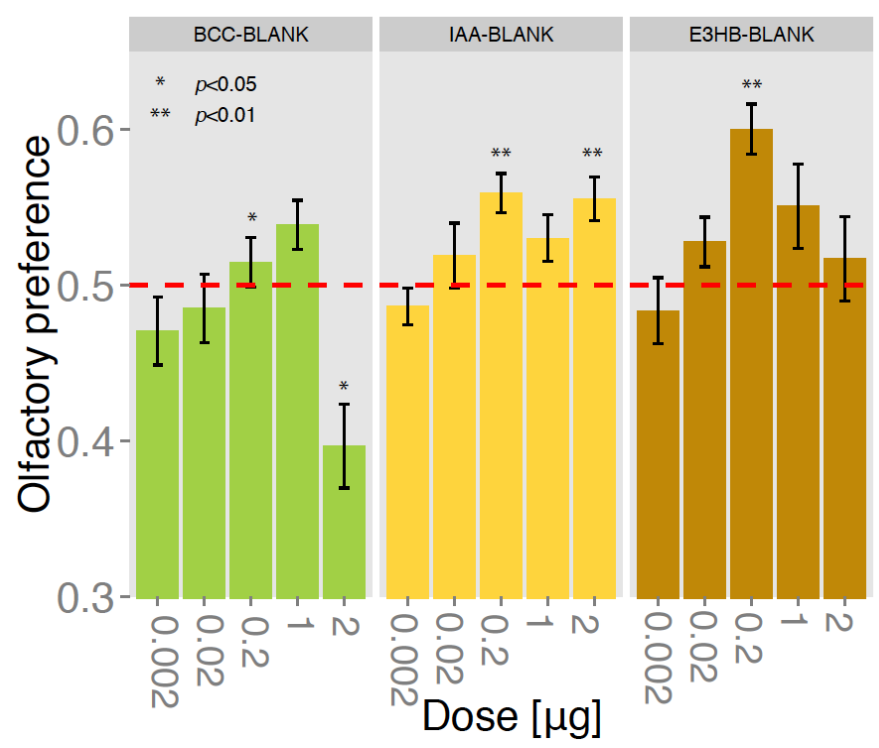

Figure 3.

Olfactory preference (proportion of flies that chose the arm with the odorant $v s$. the arm with the blank stimulus) in the Odor vs. Blank experiment as a function of Stimulus and Dose. Mean \pm SEM are presented for each compound and dose tested. BCC (green) is the leaf compound, IAA (yellow) the ripening fruit compound, E3HB (brown) the overripe fruit compound. Data above the chance level (red dashed line) indicate a preference towards the odorant, whereas data below the chance level indicate preferences for the blank stimulus $(20 \mu \mathrm{l}$ of water).

For IAA, doses of $0.2 \mu \mathrm{g}$ and $2 \mu \mathrm{g}$ were attractive and dose $1 \mu \mathrm{g}$ showed a trend in the same direction. Overall, we observed attraction for IAA compared to Blank $\left(t_{152}=4.53, p<0.001\right.$, Mean $=0.533$, SEM = 0.007).

For E3HB, a dose of $0.21 \mu \mathrm{g}$ was highly attractive, while doses of $1 \mu \mathrm{g}$ and $2 \mu \mathrm{g}$ showed a trend towards attraction for the odorant. Overall, we observed attraction for E3HB compared to the control stimulus $\left(t_{119}=4.52, p<0.001\right.$, Mean $=0.54$, SEM $\left.=0.009\right)$.

\begin{tabular}{|l|c|c|c|}
\hline Compound & Dose $[\mu \mathrm{g}]$ & $t_{d f}$ & $p$ \\
\hline BCC & 0.002 & $t_{22}=-1.35$ & 0.19 \\
\hline BCC & 0.02 & $t_{19}=-0.67$ & 0.51 \\
\hline BCC & 0.2 & $t_{27}=0.93$ & 0.36 \\
\hline BCC & 1 & $t_{37}=2.47$ & $0.018^{*}$ \\
\hline BCC & 2 & $t_{20}=-3.84$ & $0.0009^{*}$ \\
\hline IAA & 0.002 & $t_{19}=-1.13$ & 0.27 \\
\hline IAA & 0.02 & $t_{30}=0.92$ & 0.36 \\
\hline IAA & 0.2 & $t_{29}=4.68$ & $<0.001^{*}$ \\
\hline IAA & 1 & $t_{41}=2.03$ & 0.049 \\
\hline IAA & 2 & $t_{29}=3.99$ & $<0.001^{*}$ \\
\hline E3HB & 0.002 & $t_{19}=-0.77$ & 0.45 \\
\hline E3HB & 0.02 & $t_{29}=1.75$ & 0.091 \\
\hline E3HB & 0.2 & $t_{29}=6.19$ & $<0.001^{*}$ \\
\hline E3HB & 1 & $t_{19}=1.89$ & 0.074 \\
\hline E3HB & 2 & $t_{19}=1.31$ & 0.21 \\
\hline
\end{tabular}

\section{Table 2.}

Results of one-sample $t$-tests for the olfactory preference in the Odor vs. Blank experiment for each stimulus and dose vs. the chance level $(0.5)$. Significance is tested for each stimulus with Bonferroni-Holm correction $\left(^{*}=\right.$ significant). 
As for the choice ratio, we performed ANOVA on arcsin-transformed data, given that after this transformation data were not significantly different from the normal distribution (Shapiro test: $W=0.994, p=0.14)$. We found a significant effect of Stimulus $\left(F_{2,391}=3.36, p=\right.$ 0.036), a significant effect of Dose $\left(F_{4,391}=4.58, p=0.001\right)$, and a significant interaction Stimulus $\times$ Dose $\left(F_{8,391}=10.05, p=0.026\right)$, see Figure 4 .

For each compound, we used independent one-sample $t$-tests to assess whether flies were significantly attracted by that odorant compared to the baseline, which we established using the average choice ratio observed in the Blank vs. Blank control assays (Mean $=0.71$ ). Overall, each compound attracted more flies than control (BCC: $t_{131}=4.83, p<0.001$, Mean $=0.87$, $\mathrm{SEM}=0.016$; IAA: $t_{152}=6.53, p<0.001$, Mean $=0.88, \mathrm{SEM}=0.013$; E3HB: $t_{120}=9.06$, Mean $=$ 0.92, SEM $=0.014$ ).

ANOVA of the BCC data revealed only a trend for a Dose effect $\left(F_{4,127}=2.22, p=0.07\right)$, but exploratory analysis of the data (Fig. 4) showed that the leaf compound had a peak of choices at dose $0.02 \mu \mathrm{g}$ and a progressive decrease of choices towards higher doses. A different scenario was found for IAA and E3HB, which showed a significant effect of Dose (IAA: $F_{4,148}=$ 3.53, $p=0.009$; E3HB: $F_{4,116}=8.66, p<0.001$ ) with an increase towards higher doses.

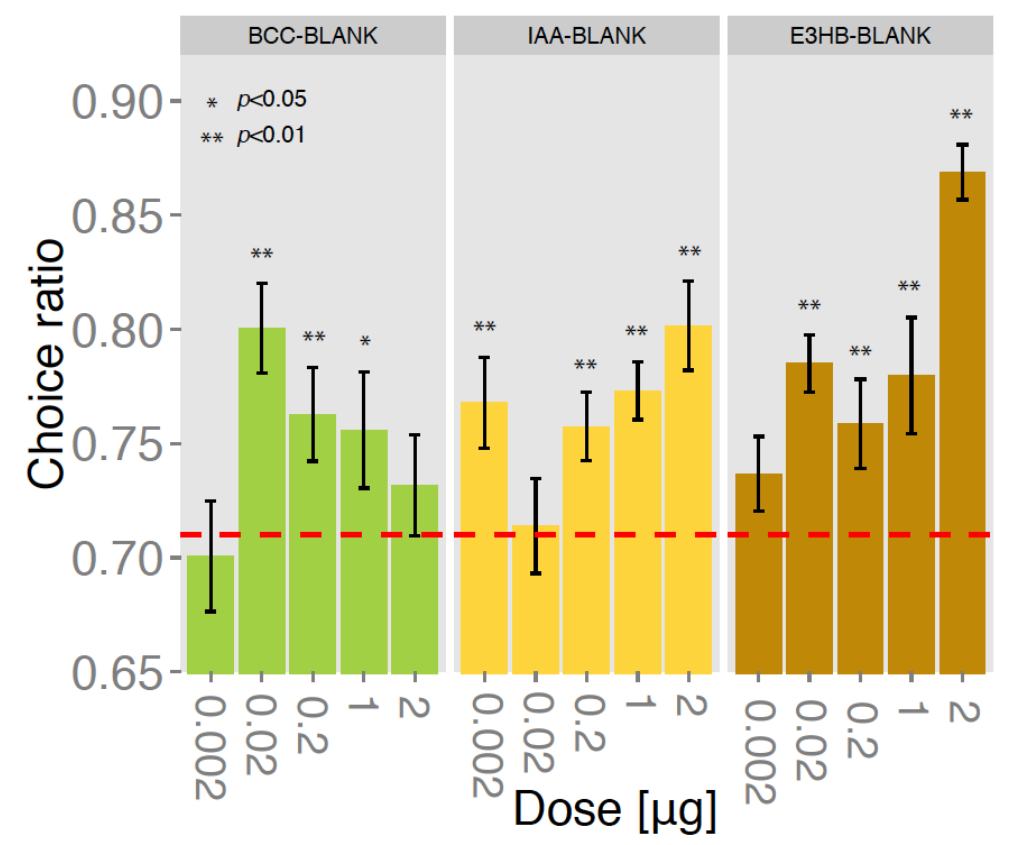

Figure 4 .

Proportion of flies that entered the odor chambers instead of remaining in the starting chamber (choice ratio) in the Odor $v s$. Blank experiment, as a function of Stimulus and Dose. Mean \pm SEM are presented for each compound and all doses tested. BCC (green) is the leaf compound, IAA (yellow) the ripening fruit compound, and E3HB (brown) the fermented fruit compound. The baseline for the choice ratio (red dashed line) was established measuring the number of flies that entered the odor chambers when only the control compound ( $20 \mu \mathrm{l}$ of water) was located in the odor chambers.

\begin{tabular}{|l|r|c|c|}
\hline Compound & Dose $[\mu \mathrm{g}]$ & $t_{d f}$ & $p$ \\
\hline BCC & 0.002 & $t_{22}=-0.005$ & 1 \\
\hline BCC & 0.02 & $t_{19}=4.74$ & $<0.001^{*}$ \\
\hline BCC & 0.2 & $t_{27}=2.94$ & $0.007^{*}$ \\
\hline BCC & 1 & $t_{37}=2.60$ & $0.013^{*}$ \\
\hline BCC & 2 & $t_{22}=1.35$ & 0.19 \\
\hline IAA & 0.002 & $t_{19}=2.91$ & $0.009^{*}$ \\
\hline IAA & 0.02 & $t_{30}=0.65$ & 0.52 \\
\hline
\end{tabular}




\begin{tabular}{|l|r|c|c|}
\hline IAA & 0.2 & $t_{29}=3.46$ & $0.002^{*}$ \\
\hline IAA & 1 & $t_{41}=5.21$ & $<0.001^{*}$ \\
\hline IAA & 2 & $t_{29}=4.62$ & $<0.001^{*}$ \\
\hline E3HB & 0.002 & $t_{19}=1.86$ & 0.079 \\
\hline E3HB & 0.02 & $t_{29}=5.39$ & $<0.001^{*}$ \\
\hline E3HB & 0.2 & $t_{29}=3.04$ & $0.005^{*}$ \\
\hline E3HB & 1 & $t_{19}=3.15$ & $0.005^{*}$ \\
\hline E3HB & 2 & $t_{20}=10.63$ & $<0.001^{*}$ \\
\hline
\end{tabular}

Table 3.

Results of one-sample $t$-tests for the choice ratio in the Odor vs. Blank experiment, for each stimulus and dose $v s$. the baseline. Significance is tested for each stimulus with Bonferroni-Holm correction $(*=$ significant). Data have been arcsin transformed before analysis.

\section{T-maze behavioral assays: Odor vs. Odor experiment}

For all odorants pairs (IAA-BCC, E3HB-BCC, E3HB-IAA) we tested the olfactory preference for the odorant associated with the later stage of fruit maturation and the overall choice ratio at the three highest doses $(0.2,1,2 \mu \mathrm{g})$.

The ANOVA on the olfactory preference showed a significant main effect of Stimulus $\left(F_{2,249}=\right.$ $21.3, p<0.001)$ and Dose $\left(F_{2,249}=5.05, p=0.007\right)$ and a significant interaction Stimulus $\times$ Dose $\left(F_{4,249}=3.87, p=0.0045\right)$, see Figure 5 .

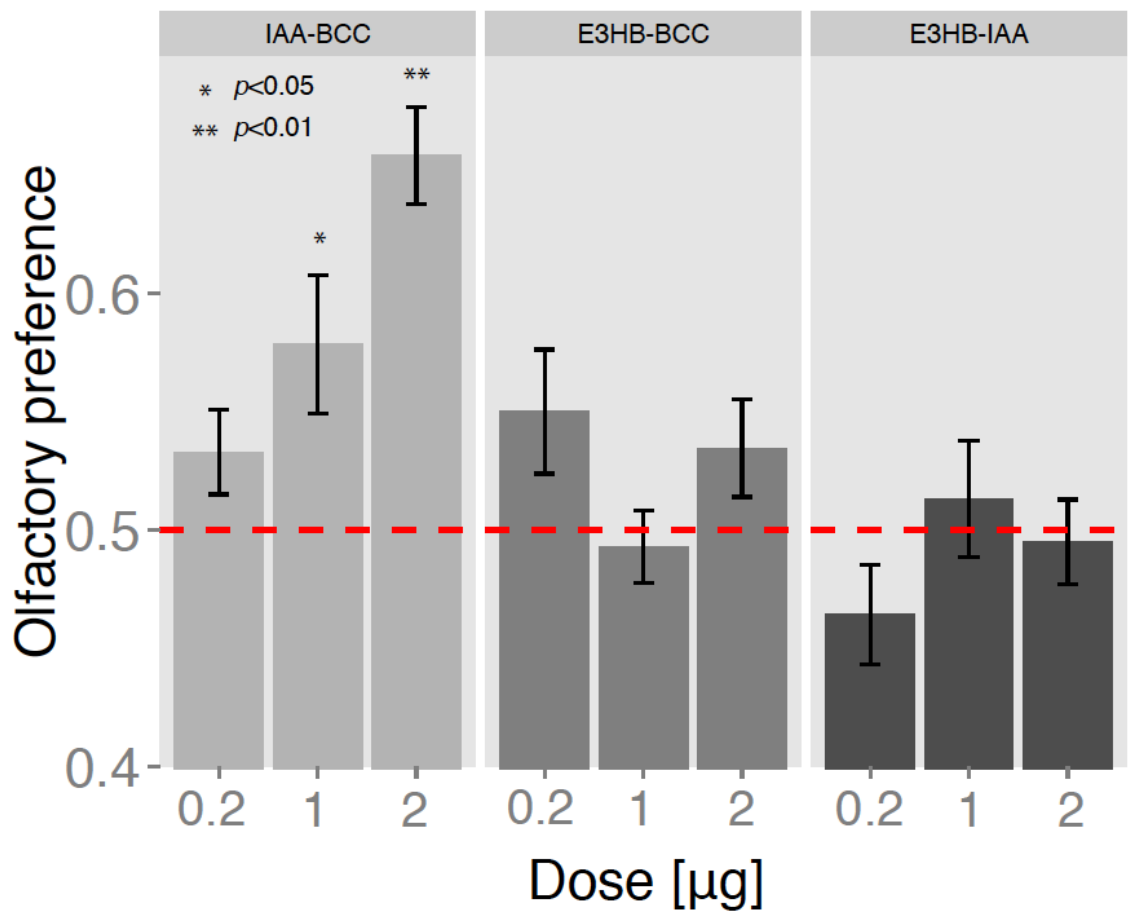

Figure 5.

Preferences for odorants presented in the Odor vs. Odor experiment as a function of Stimulus and Dose. Data below 0.5 (red dashed line) indicate a preference for the odorant that in each test corresponded to the earlier stage of maturation, whereas data above the chance level indicate a preference for the odorant corresponding to the later stage of maturation. Mean \pm SEM are presented for each pair of odorants and all tested doses. The pair IAA (ripening fruit compound)-BCC (leaf compound) is presented in the left panel, the pair E3HB (fermented fruit compound)-BCC in the central panel, the pair E3HB-IAA in the right panel. 


\begin{tabular}{|l|r|c|c|}
\hline Compounds & $\begin{array}{c}\text { Dose of each } \\
\text { compound }[\mu \mathrm{g}]\end{array}$ & $t_{d f}$ & $p$ \\
\hline IAA-BCC & 0.2 & $t_{22}=1.84$ & 0.08 \\
\hline IAA-BCC & 1 & $t_{19}=2.69$ & $0.015^{*}$ \\
\hline IAA-BCC & 2 & $t_{38}=7.74$ & $<0.001^{*}$ \\
\hline E3HB-BCC & 0.2 & $t_{19}=1.91$ & 0.071 \\
\hline E3HB-BCC & 1 & $t_{42}=-0.46$ & 0.65 \\
\hline E3HB-BCC & 2 & $t_{38}=1.68$ & 0.10 \\
\hline E3HB-IAA & 0.2 & $t_{20}=-1.70$ & 0.11 \\
\hline E3HB-IAA & 1 & $t_{21}=0.54$ & 0.60 \\
\hline E3HB-IAA & 2 & $t_{30}=-0.28$ & 0.79 \\
\hline
\end{tabular}

Table 4. Results of one-sample $t$-tests for the olfactory preference in the Odor $v s$. Odor experiment for each stimulus and dose vs. the chance level (0.5). Significance is tested with Bonferroni-Holm correction $\left(^{*}=\right.$ significant).

Considering the average of all doses, in post-hoc $t$-tests with Bonferroni-Holm correction (from alpha $<0.05$, see Table 4) we observed a strong preference for the ripening fruit compound IAA vs. the leaf compound BCC $\left(t_{81}=7.27, p<0.001\right.$, Mean $=0.60$, SEM $\left.=0.014\right)$ with a rapid increase of the preference at higher doses $\left(F_{2,79}=8.69, p<0.001\right)$. On the contrary, for the fermented fruit compound E3HB vs. the leaf compound BCC we documented only a trend towards the later maturation compound $\mathrm{E} 3 \mathrm{HB}\left(t_{101}=1.73\right.$, Mean $=0.52, \mathrm{SEM}=$ $0.012)$ and no significant effect of Dose $\left(F_{2,99}=2.17, p=0.12\right)$. There was no significant preference between the odorants corresponding to the later stages of fruit maturation IAA and E3HB $\left(t_{73}=-0.69, p=0.50\right.$, Mean $\left.=0.50, \mathrm{SEM}=0.012\right)$ and no Dose effect $\left(F_{2,71}=1.23, p=\right.$ $0.3)$.

As for the choice ratio (proportion of flies that entered the odor chambers) we performed statistical parametric analyzes on the arcsin-transformed data, given that after this transformation data were not significantly different from the normal distribution (Shapiro test, $W=0.99, p=0.11$ ). For this measure we observed a significant main effect of Stimulus $\left(F_{2,249}=6.15, p=0.002\right)$, no significant effect of Dose $\left(F_{2,249}=0.87, p=0.42\right)$, and a significant interaction Stimulus $\times$ Dose $\left(F_{4,249}=3.55, p=0.008\right)$, see Figure 6 . Using one-sample $t$-tests with Bonferroni-Holm correction, all pairs of odorants (IAA-BCC, E3HB-BCC, E3HB-IAA) were significantly more attractive than the Blank $v s$. Blank control (IAA-BCC: $t_{81}=9.24, p<0.001$; E3HB-BCC: $t_{101}=13.9, p<0.001$; E3HB-IAA: $t_{73}=14.6, p<0.001$, see Table 5 ). The pair of odorants associated with earlier stages of fruit maturation (green leaf and ripening fruit) elicited significantly less choices than the other odorant pairs (IAA-BCC $v s$. E3HB-BCC: $t_{169.5}=$ 1.98, $p=0.049$; IAA-BCC $v s$. E3HB-IAA: $t_{153.9}=3.43, p<0.001$ ), and we observed a trend for the odorant pair corresponding to ripening and fermented fruit to elicit more choices compared to the leaf and fermented odorant (E3HB-BCC vs. E3HB-IAA: $t=-1.67, d f=161.09, p=0.096$ ), which is an intermediate combination. 


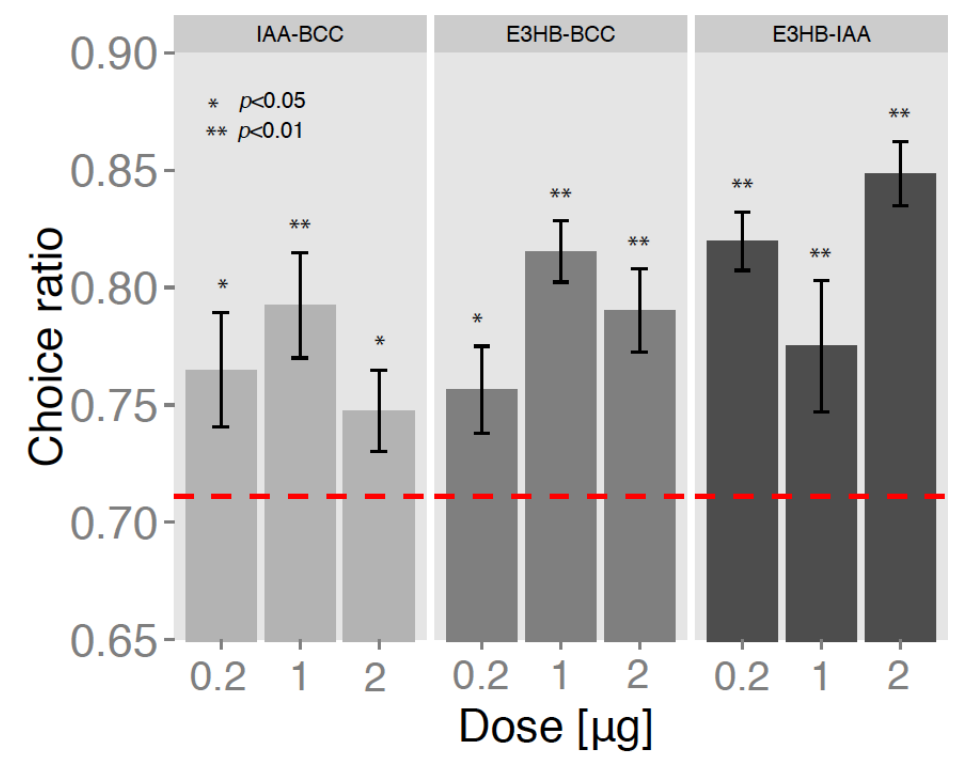

Figure 6.

Proportion of flies that entered the odor chambers instead of remaining in the starting chamber (choice ratio) in the Odor vs. Odor experiment as a function of Stimulus and Dose. Mean \pm SEM are presented for each compound and dose tested. The pair IAA (ripening fruit compound)-BCC (leaf compound) is presented in the left panel, the pair E3HB (fermented fruit compound)-BCC in the central panel, the pair E3HB-IAA in the right panel. The baseline for the choice ratio (red dashed line) was established by measuring the number flies that entered the odor chambers when only the control compound (20 $\mu$ l of water) was located in the odor chambers.

\begin{tabular}{|l|r|c|c|}
\hline Compounds & $\begin{array}{c}\text { Dose of each } \\
\text { compound }[\mu \mathrm{g}]\end{array}$ & & $p$ \\
\hline IAA-BCC & 0.2 & $t_{22}=2.65$ & 0.015 \\
\hline IAA-BCC & 1 & $t_{19}=3.76$ & $0.001^{*}$ \\
\hline IAA-BCC & 2 & $t_{38}=2.65$ & 0.012 \\
\hline E3HB-BCC & 0.2 & $t_{19}=2.69$ & 0.015 \\
\hline E3HB-BCC & 1 & $t_{42}=8.05$ & $<0.001^{*}$ \\
\hline E3HB-BCC & 2 & $t_{38}=4.82$ & $<0.001^{*}$ \\
\hline E3HB-IAA & 0.2 & $t_{20}=8.45$ & $<0.0001^{*}$ \\
\hline E3HB-IAA & 1 & $t_{21}=2.91$ & $0.008^{*}$ \\
\hline E3HB-IAA & 2 & $t_{30}=9.16$ & $<0.001^{*}$ \\
\hline
\end{tabular}

Table 5 .

Results of one-sample $t$-tests for the choice ratio in the Odor vs. Odor experiment, for each stimulus and dose $v s$. the baseline. Significance is tested with Bonferroni-Holm correction $*^{*}=$ significant) of arcsin-transformed data.

\section{T-maze behavioral assays: Same odors experiment}

In the Odor vs. Odor experiment, two different odorants were presented simultaneously. In this way the overall amount of odorants present in the apparatus is doubled compared to the Odor $v s$. Blank experiment. To investigate how the choice ratio is affected by the mixture of two different odorants and by the increase of odorants in the apparatus, we added a control experiment in which we presented two identical odorants (Same odors experiment) in the odor chambers: BCC-BCC, IAA-IAA and E3HB-E3HB, presented at dose 0.002, 0.02, 0.2, 1, and $2 \mu \mathrm{g}$. Exploratory data analyses revealed one outlier, that was removed for further analyses (its inclusion/exclusion did not affect significance). We ran an ANOVA on the arcsintransformed data (after this transformation, data were not significantly different from the normal distribution: Shapiro test, $W=0.99, p=0.09$ ). When analyzing all five doses we 
detected a main effect of Stimulus $\left(\mathrm{F}_{2,284}=3.68, p=0.03\right)$, no main effect of Dose $\left(\mathrm{F}_{4,284}=0.93\right.$, $p=0.45)$, and a trend for a Stimulus $\times$ Dose interaction $\left(\mathrm{F}_{8,284}=9.04, p=0.08\right)$, see Fig. 7 for the modulation induced by different stimuli and doses. Table 6 shows the results of the $t$-tests of the choice ratio for each stimulus and dose $v s$. the baseline level.

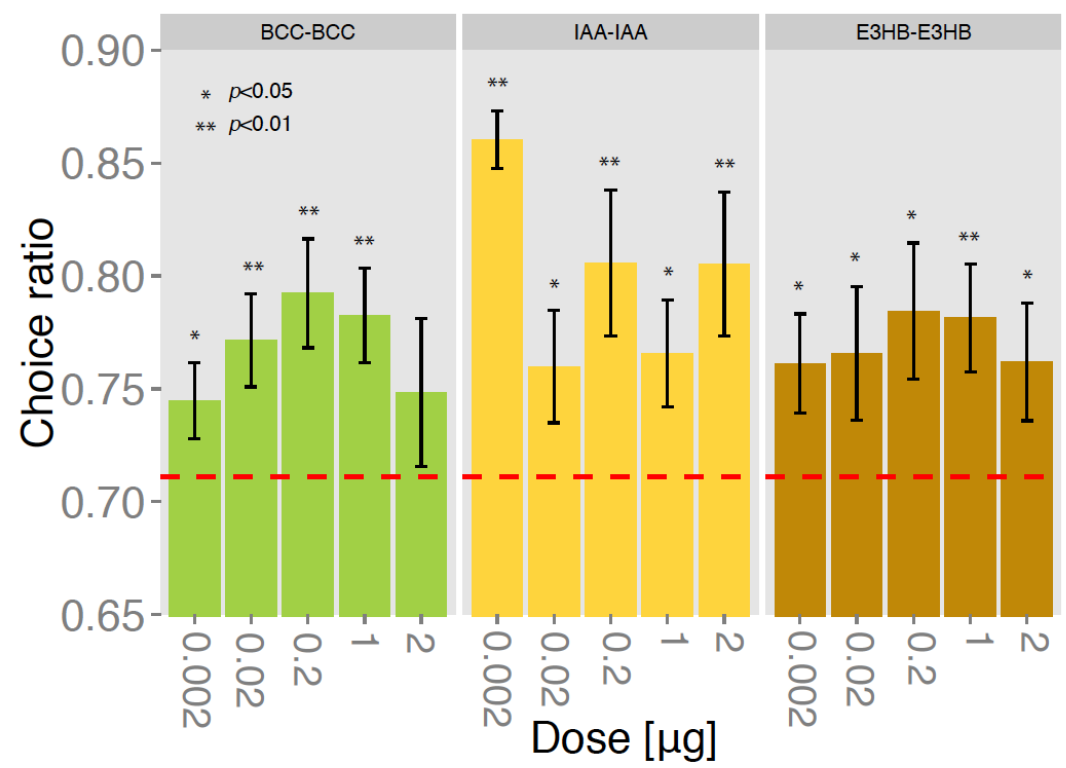

Figure 7.

Proportion of flies that entered the odor chambers instead of remaining in the starting chamber (choice ratio) in the Same odors experiment as a function of Stimulus and Dose. Mean \pm SEM are presented for each compound and dose tested. The pair BCC-BCC (leaf odorant) is presented in the left panel, the pair IAA-IAA (ripening fruit compound) in the central panel, the pair E3HB-E3HB (fermenting fruit compound) in the right. The baseline for the choice ratio (red dashed line) was established by measuring the number flies that entered the odor chambers when only the control compound ( $20 \mu \mathrm{l}$ of water) was located in the odor chambers.

\begin{tabular}{|l|r|c|c|}
\hline Compounds & $\begin{array}{l}\text { Dose of each } \\
\text { compound }[\mu \mathrm{g}]\end{array}$ & \multicolumn{1}{c|}{$t_{d f}$} & $p$ \\
\hline BCC-BCC & 0.002 & $t_{19}=2.31$ & 0.03 \\
\hline BCC-BCC & 0.02 & $t_{19}=3.14$ & $0.005^{*}$ \\
\hline BCC-BCC & 0.2 & $t_{19}=3.52$ & $0.002^{*}$ \\
\hline BCC-BCC & 1 & $t_{19}=3.65$ & $0.002^{*}$ \\
\hline BCC-BCC & 2 & $t_{19}=1.67$ & 0.11 \\
\hline IAA-IAA & 0.002 & $t_{19}=10.24$ & $<0.001^{*}$ \\
\hline IAA-IAA & 0.02 & $t_{19}=2.37$ & 0.029 \\
\hline IAA-IAA & 0.2 & $t_{19}=3.48$ & $0.002^{*}$ \\
\hline IAA-IAA & 1 & $t_{19}=2.76$ & 0.012 \\
\hline IAA-IAA & 2 & $t_{18}=4.80$ & $<0.001^{*}$ \\
\hline E3HB-E3HB & 0.002 & $t_{19}=2.71$ & 0.014 \\
\hline E3HB-E3HB & 0.02 & $t_{19}=2.34$ & 0.030 \\
\hline E3HB-E3HB & 0.2 & $t_{19}=2.81$ & 0.011 \\
\hline E3HB-E3HB & 1 & $t_{19}=3.20$ & $0.005^{*}$ \\
\hline E3HB-E3HB & 2 & $t_{19}=2.38$ & 0.03 \\
\hline
\end{tabular}

Table 6.

Results of one-sample $t$-tests for the choice ratio in the Same odors experiment, for each stimulus and dose vs. the baseline. Significance is tested for each stimulus with Bonferroni-Holm correction $(*=$ significant) of arcsintransformed data. 


\section{T-maze behavioral assays: Two odors vs. Blank experiment}

To investigate the effect of co-occurrent sources of odorants, we located two odorants in the same odor chamber and a blank stimulus in the other chamber. We tested the olfactory preference and choice ratio for odorant pairs (IAA and BCC, E3HB and BCC, E3HB and IAA) vs. Blank at dose $0.2,1$ and $2 \mu \mathrm{g}$. For the olfactory preference the ANOVA showed a significant main effect of Stimulus $\left(F_{2,169}=8.82, p<0.001\right)$ and Dose $\left(F_{2,169}=12.98, p<0.001\right)$ and a significant interaction Stimulus $\times$ Dose $\left(F_{4,169}=4.36, p=0.002\right)$, see Fig. 8. For the IAA and BCC $v s$. Blank condition there was no significant difference between doses $\left(\mathrm{F}_{2,56}=0.49, p=0.16\right)$, and overall we detected a significant preference for the odorants $\left(t_{58}=5.06, p<0.001\right)$. For the E3HB and BCC vs. Blank condition we observed a significant effect of Dose $\left(\mathrm{F}_{2,55}=8.96, p<\right.$ 0.001), with an opposite trend for the highest doses (see Table 7). For the E3HB and IAA vs. Blank condition we observed a significant effect of Dose, with only low and intermediate doses eliciting attraction (see Table 7). In the Odor vs. Odor experiment we observed the highest choice ratio for the E3HB vs. IAA pairing. To check whether the same pattern was present also with co-occurrent sources of odorants, we contrasted the choice ratio of the three pairs stimuli overall: in the presence of E3HB and IAA stimulus, flies responded significantly more than with IAA and BCC $\left(\mathrm{t}_{115.3}=\right.$ $2.54, p=0.01)$ and E3HB and BCC $\left(\mathrm{t}_{116.3}=3.48, p<0.001\right)$, confirming the pattern observed in the Odor $v s$. Odor experiment.

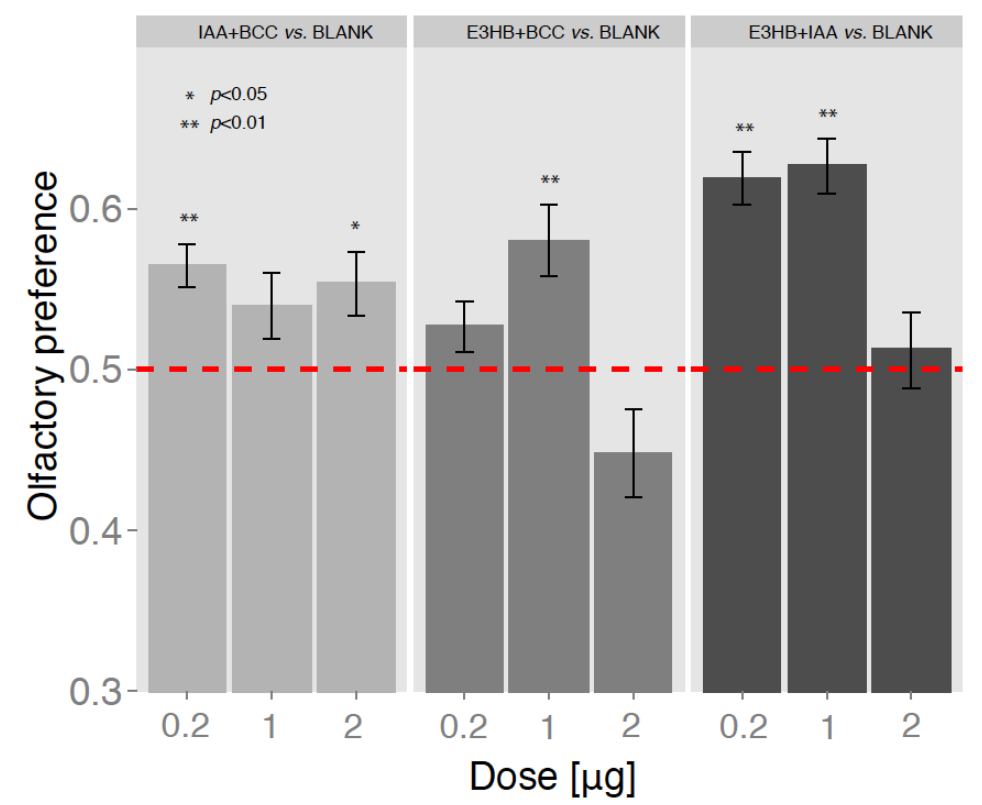

Figure 8.

Preferences for pairs of compounds presented in the Two odors vs. Blank experiment as a function of Stimulus pair and Dose. Data below 0.5 (red dashed line) indicate a preference for Blank, whereas data above the chance level indicate a preference for the odorants pair presented. Mean \pm SEM are presented for each pair of odorants and all dose tested. The pair IAA (ripening fruit compound)-BCC (leaf compound) is presented in the left panel, the pair E3HB (fermented fruit compound)-BCC in the central panel, the pair E3HB-IAA in the right panel. 


\begin{tabular}{|l|r|c|c|}
\hline Compounds & $\begin{array}{c}\text { Dose of each } \\
\text { compound }[\mu \mathrm{g}]\end{array}$ & & $p$ \\
\hline IAA and BCC & 0.2 & $t_{19}=4.84$ & $<0.001^{*}$ \\
\hline IAA and BCC & 1 & $t_{19}=1.94$ & 0.07 \\
\hline IAA and BCC & 2 & $t_{18}=2.68$ & 0.015 \\
\hline E3HB and BCC & 0.2 & $t_{19}=1.66$ & 0.11 \\
\hline E3HB and BCC & 1 & $t_{19}=3.60$ & $0.002^{*}$ \\
\hline E3HB and BCC & 2 & $t_{17}=-1.92$ & 0.07 \\
\hline E3HB and IAA & 0.2 & $t_{22}=7.20$ & $<0.001^{*}$ \\
\hline E3HB and IAA & 1 & $t_{18}=7.48$ & $<0.001^{*}$ \\
\hline E3HB and IAA & 2 & $t_{18}=0.51$ & 0.61 \\
\hline
\end{tabular}

\section{Table 7.}

Results of one-sample $t$-tests for the olfactory preference in the Two odors $v s$. Blank experiment for each stimulus and dose $v s$. the chance level (0.5). Significance is tested with Bonferroni-Holm correction $\left(^{*}=\right.$ significant).

Exploratory data analyses revealed two outliers that were removed for further analyses (the overall results did not change including or excluding these observations). We performed ANOVA on arcsin-transformed data, given that after this transformation data were not significantly different from the normal distribution (Shapiro test: $W=0.987, p=0.09$ ), using Stimulus (IAA+BCC vs. BLANK, E3HB+BCC vs. BLANK, E3HB+IAA vs. BLANK) and Dose (0.2, 1, $2 \mu \mathrm{g}$ for each compound) as independent variables. We observed a significant effect of Stimulus $\left(F_{2,173}=5.18, p=0.007\right)$, a significant effect of Dose $\left(F_{2,173}=5.39, p=0.005\right)$, and a significant interaction Stimulus $\times$ Dose $\left(F_{4,173}=5.17, p<0.001\right)$, see Figure 9 . To investigate the difference in choice ratio between the Two odors $v s$. Blank experiment and the Odor vs. Odor experiment, we conducted post-hoc $t$-tests with Bonferroni-Holm correction for different odorant pairs and concentrations presented in these two experiments (from $\alpha<0.05$, see Table 7). As Figure 8 shows, for all conditions there was a trend for higher choice rate in the Two odors vs. Blank experiment, although not always significant. This implies a greater effectiveness of odorant sources with multiple odorants in attracting Drosophila melanogaster. 


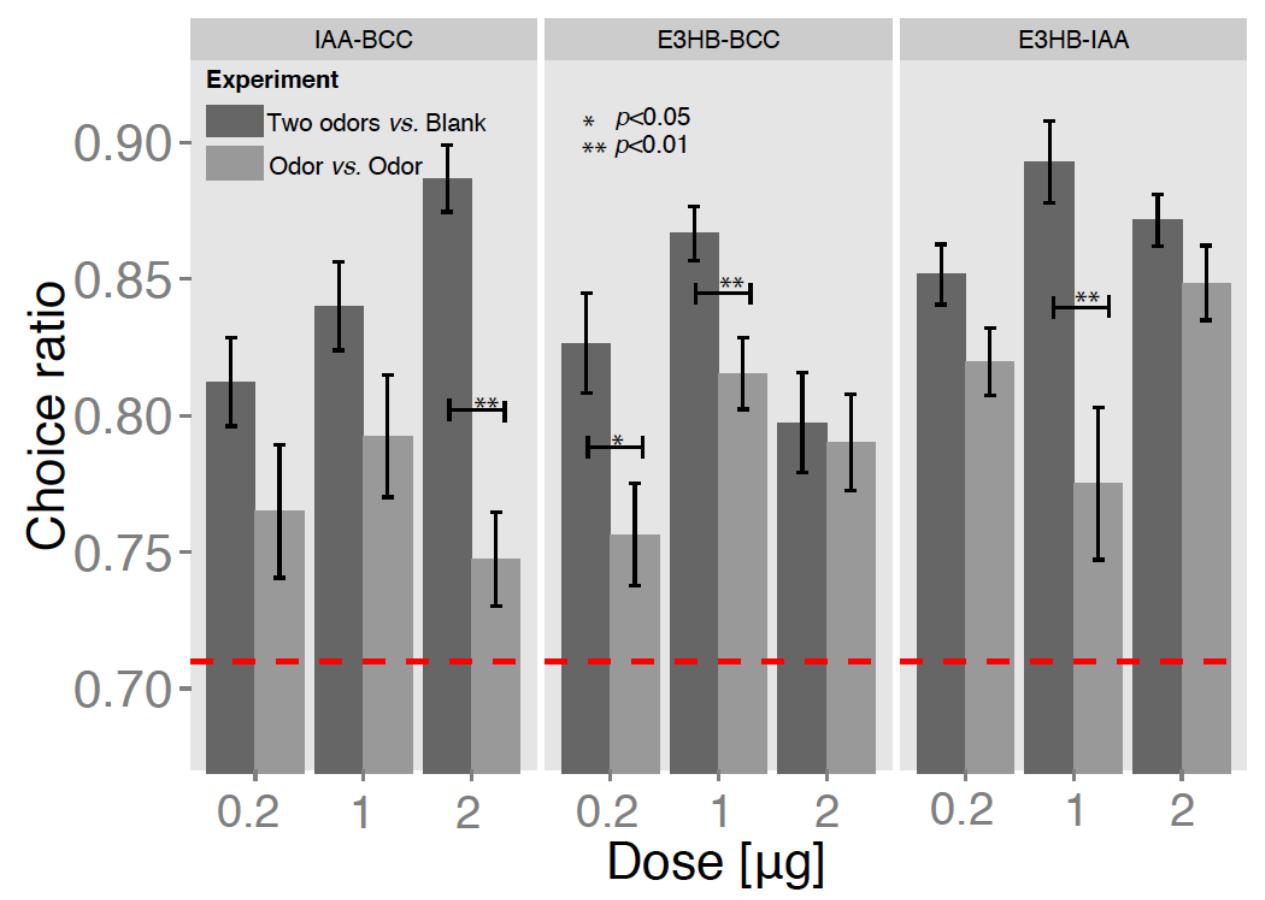

Figure 9.

Proportion of flies that entered the odor chambers instead of remaining in the starting chamber (choice ratio) in the Two odors vs. Blank experiment (dark grey bars) and in the Odor vs. Odor experiment (light grey bars) as a function of Stimulus and Dose. Mean \pm SEM are presented for each compound and dose tested. The pair IAA (ripening fruit compound)-BCC (leaf compound) is presented in the left panel, the pair E3HB (fermented fruit compound)-BCC in the central panel, the pair E3HB-IAA in the right panel. The baseline for the choice ratio (red dashed line) was established by measuring the number flies that entered the odor chambers when only the control compound ( $20 \mu \mathrm{l}$ of water) was located in the odor chambers.

\begin{tabular}{|l|r|c|c|}
\hline Compounds & $\begin{array}{c}\text { Dose of each } \\
\text { compound }[\mu \mathrm{g}]\end{array}$ & $t_{d f}$ & $p$ \\
\hline IAA and BCC & 0.2 & $t_{37}=1.62$ & 0.11 \\
\hline IAA and BCC & 1 & $t_{34.6}=1.72$ & 0.09 \\
\hline IAA and BCC & 2 & $t_{57}=6.58$ & $<0.001^{*}$ \\
\hline E3HB and BCC & 0.2 & $t_{38}=2.69$ & 0.01 \\
\hline E3HB and BCC & 1 & $t_{60.3}=3.15$ & $0.003^{*}$ \\
\hline E3HB and BCC & 2 & $t_{49.2}=0.28$ & 0.78 \\
\hline E3HB and IAA & 0.2 & $t_{41.6}=1.93$ & 0.06 \\
\hline E3HB and IAA & 1 & $t_{32}=3.72$ & $<0.001^{*}$ \\
\hline E3HB and IAA & 2 & $t_{48.3}=1.38$ & 0.17 \\
\hline
\end{tabular}

\section{Table 8 .}

Results of independent $t$-tests for the choice ratio in the Two odors vs. Blank experiment against the Odor vs. Odor experiment, for each stimulus and dose. Significance is tested with Bonferroni-Holm correction $\left(^{*}=\right.$ significant).

\section{DISCUSSION}

Fruit flies effectively use odors to find food [2,3,32,34] and mates [24,35]. The observation that in D. melanogaster most olfactory receptors are responsive to fruit odors [3] is consistent with the ecology of a species that feeds, mates and oviposits on overripe fruit. While many studies have investigated the responses of fruit flies to compounds associated with fruit fermentation $[19,20,36]$, including yeast $[32,37]$, less attention has been devoted to compounds associated with the early stages of fruit maturation. This information is important 
to understand the natural history of drosophilids and how they can occupy different ecological niches [38]. For instance, Drosophila species of the "cosmopolitan guild" [39] differ in the time of colonization of rotten fruit. The first species to colonize rotting fruit is $D$. simulans, which approaches fruit when few volatiles have been produced by fermentation, followed by D. melanogaster and D. immigrans, and then by the other species Drosophila hydei and Drosophila busckii [40,41]. Beside fermenting fruit, other parts of plants and maturation stages are attractive for certain Drosophila species and reflect different olfactory responses [36]: D. virilis [42] and D. pseudoobscura [43] are attracted by the tree sap, D. obscura and D. subobscura feed on leaves within the canopy [44,45], D. mojavensis is specialized on photosynthetic tissue of fermenting cactus [26], D. suzukii has evolved a segmented ovipositor that allows to cut the skin of small fruits and preferentially oviposits on ripening fruit [46]. Keesey et al. [17] have compared three Drosophila species, finding that while D. suzukii, that oviposits on fresh fruit, responded to the leaf compound $\beta$-cyclocitral both at the electrophysiological (responses of the olfactory sensory neuron ab3A) and behavioral level (trap assays), D. melanogaster and D. biarmipes, that do not oviposit on fresh ripe fruit, did not. Based on this negative evidence Keesey et al. [17] hypothesized that the ecological shift of D. suzukii towards fresh fruit might be sustained by a sensory specialization for odors associated with early stages of fruit maturation.

To investigate responses of a D. melanogaster population for compounds associated to different stages of fruit maturation, we assayed sensory and behavioral responses of the DGRP D. melanogaster population [29] to synthetic compounds found in leaves, ripening fruit and fermented media, that we used as "placeholders" for different phonological stages [1720,36]. The use of synthetic compounds known to be present in different stages of fruit maturation enabled us to precisely control the amount of each odorant and combinations of odorants presented, while maintaining a connection to the media important for Drosophila in the wild.

Flies' sensory responses were investigated using electroantennograms, which are an overall measure for the response of olfactory receptor neurons present on the antenna. Differently from previous reports [17], we found that D. melanogaster can indeed sense the leaf compound $\beta$-cyclocitral (BCC), although flies are less sensitive to low concentrations compared to compounds associated with later maturation stages. It is possible that our positive results depend on the fact that electroantennography - on the contrary to the single sensillum recording technique used in the work of Keesey et al. [17] - shows the responses of several types of olfactory neurons on the antenna. Other volatiles known to be associated to unripen fruit such as $\mathrm{CO}_{2}$ [47], or to the green leaf stage such as 1-hexanol [20] and E2hexenal $[48,49]$, are aversive for D. melanogaster.

Via the odorant concentration difference detected by the two antennae, flies can orient to a still chemical gradient, locate an odor source [50] and approach it. To assess this complex behavioral response we performed T-maze tests on representative odorants for each maturation stage: BCC for the leaf stage, isoamyl acetate (IAA) for the ripening fruit stage, and ethyl 3-hydroxybutyrate (E3HB) for the fermented fruit stage. Differently from other studies [e.g. 18 for individually tested flies], we assayed groups $(n=100)$ of fruit flies of both sexes, with different haplotypes, to have a representative of our population. Drosophila melanogaster aggregates on food patches and it has been shown that food choices and aggregation can be affected by group size $[51,52]$. Hence, group testing can mirror ecologically relevant dynamics, possibly more than individual testing. When facing the choice between each individual compound and pure water (blank control) (see Odor vs. Blank experiment), flies were attracted by the fresh fruit and fermented fruit compound at different doses, with a peak at higher doses for the ripe fruit compound, and at intermediate doses for the fermented compound. Instead, flies were attracted by the leaf 
compound at intermediate dose $(1 \mu \mathrm{g})$ but were strongly repelled by the same compound at the highest dose $(2 \mu \mathrm{g})$. Consistently with this outcome, flies progressively decreased the choice ratio from the intermediate to the highest dose for the leaf compound, while they increased their choice ratio at higher doses for the other compounds. This suggests that, when a leaf compound is presented in isolation, it can attract $D$. melanogaster only at intermediate/low concentrations before becoming aversive, while compounds associated with later stages of fruit maturation effectively attract flies at a broader range of concentrations. The DGRP drosophila population of isogenic lines [29] is a good candidate to carry on further studies to clarify how differences at the peripheral and central level can determine these outcomes.

When testing flies with pairs of odorants (Odor vs. Odor experiment) we did not have a precise control on the mixture of the two odorants in the apparatus. In spite of this we observed that, as expected, when pairing the later maturation stage compounds (E3HB vs. IAA) flies were more attracted compared to other pairs of stimuli, although we did not observe any significant preference for each of the two compounds. Surprisingly though, when pairing the fermented compound (E3HB) with the ripening fruit compound (IAA) and with the leaf compound (BCC) we obtained very different outcomes. While the ripening fruit compound was very much preferred over the leaf compound, we observed only a slight preference for the fermented compound over leaf. For the olfactory preference, we hence found a non-additive preference effect, since two odorants equally attractive when presented together (there was no significant preference for E3HB and IAA when they were presented in the same trial) elicited different responses when paired against another compound (IAA but not E3HB elicited a significant preference compared to BCC). We further investigated the effect of two compounds by combining together two odorants and giving a choice against blank control. In this experiment (Two odors vs. Blank) the number of flies that made a choice was significantly higher than in the Odor vs. Odor experiment, suggesting that the mixture of odorants was more effective than single odorants in attracting flies.

The results obtained with two co-occurrent odorants and contrasting two odorants suggest that olfactory stimuli are particularly attractive for D. melanogaster in specific combinations of ripening and fermented components, compared to combinations of earlier stages of maturation. It is important to consider though, that the compounds used in the present study are present in different maturation stages of the fruits and that their concentration varies between ripening and fermenting fruits. Revadi et al. [53] showed that D. suzukii significantly chose isoamyl acetate only at the concentration $10^{-2} \mu \mathrm{g} / \mu \mathrm{l}$ and associated this to the release rate from ripe fruits. In the present study we have shown that D. melanogaster is attracted to isoamyl acetate at a broad range of doses, and that this compound induced the highest choice ratio in combination with the fermented fruit compound ethyl-3-hydroxybutyrate.

Given that D. melanogaster is becoming a model not only for basic research in biology, but also for applied science [54,55], in particular due to its ecological [15] and phylogenetic [13] proximity to the pest species $D$. suzukii $[15,18,56]$, these findings are relevant to understand the species-specific adaptations of $D$. melanogaster, which will allow to plan effective strategies of pest management regarding D. suzukii.

\section{ACKNOWLEDGEMENTS}

We thank the City of Rovereto for supporting AE through the grant "Sustainable strategies against pest fruit flies: from olfactory perception to the protection of the small fruits of Trentino". We are grateful to Dr. Gianfranco Anfora for help in the choice of odorants and assistance with EAG, and to Prof. Sergio Angeli and Dr. Gianfranco Anfora for fruitful discussions throughout the project. We also thank three anonymous reviewers for their valuable comments and suggestions. 


\section{REFERENCES}

[1] S.A. Kreher, D. Mathew, J. Kim, J.R. Carlson, Translation of Sensory Input into Behavioral Output via an Olfactory System, Neuron. 59 (2008) 110-124.

doi:10.1016/j.neuron.2008.06.010.

[2] M. Knaden, A. Strutz, J. Ahsan, S. Sachse, B.S. Hansson, Spatial Representation of Odorant Valence in an Insect Brain, Cell Rep. 1 (2012) 392-399. doi:10.1016/j.celrep.2012.03.002.

[3] E.A. Hallem, J.R. Carlson, Coding of Odors by a Receptor Repertoire, Cell. 125 (2006) 143-160. doi:10.1016/j.cell.2006.01.050.

[4] S. Lebreton, P.G. Becher, B.S. Hansson, P. Witzgall, Attraction of Drosophila melanogaster males to food-related and fly odours, J. Insect Physiol. 58 (2012) 125-129. doi:10.1016/j.jinsphys.2011.10.009.

[5] P. Ramdya, P. Lichocki, S. Cruchet, L. Frisch, W. Tse, D. Floreano, et al., Mechanosensory interactions drive collective behaviour in Drosophila, Nature. 519 (2014) 233-236. doi:10.1038/nature14024.

[6] N.H. Scheidler, C. Liu, K.A. Hamby, F.G. Zalom, Z. Syed, Volatile codes: Correlation of olfactory signals and reception in Drosophila-yeast chemical communication, Sci. Rep. 5 (2015) 14059. doi:10.1038/srep14059.

[7] T. Dobzhansky, D.M. Cooper, H.J. Phaff, E.P. Knapp, H.L. Carson, Differential Attraction of Species of Drosophila to Different Species of Yeasts, Ecology. 37 (1956) 544-550. doi:10.2307/1930178.

[8] C.S. McBride, Rapid evolution of smell and taste receptor genes during host specialization in Drosophila sechellia, Proc. Natl. Acad. Sci. U. S. A. 104 (2007) 49965001. doi:10.1073/pnas.0608424104.

[9] M.C. Stensmyr, Drosophila sechellia as a model in chemosensory neuroecology, Ann. N. Y. Acad. Sci. 1170 (2009) 468-475. doi:10.1111/j.1749-6632.2009.03911.x.

[10] L. Legal, J.R. David, J.M. Jallon, Toxicity and attraction effects produced by Morinda citrifolia fruits on the Drosophila melanogaster complex of species, Chemoecology. 3 (1992) 125-129. doi:10.1007/BF01370140.

[11] G.H. Arya, M.M. Magwire, W. Huang, Y.L. Serrano-Negron, T.F.C. Mackay, R.R.H. Anholt, The Genetic Basis for Variation in Olfactory Behavior in Drosophila melanogaster, Chem. Senses. (2015) 1-11. doi:10.1093/chemse/bjv001.

[12] S.M. Rollmann, P. Wang, P. Date, S.A. West, T.F.C. Mackay, R.R.H. Anholt, Odorant receptor polymorphisms and natural variation in olfactory behavior in Drosophila melanogaster., Genetics. 186 (2010) 687-97. doi:10.1534/genetics.110.119446.

[13] L. Ometto, A. Cestaro, S. Ramasamy, A. Grassi, S. Revadi, S. Siozios, et al., Linking genomics and ecology to investigate the complex evolution of an invasive Drosophila pest., Genome Biol. Evol. 5 (2013) 745-757. doi:10.1093/gbe/evt034.

[14] G. De Ros, G. Anfora, A. Grassi, C. Ioriatti, The potential economic impact of Drosophila suzukii on small fruits production in Trentino (Italy ), 2009 (2009) 73-77.

[15] M.K. Asplen, G. Anfora, A. Biondi, D.-S. Choi, D. Chu, K.M. Daane, et al., Invasion biology of spotted wing Drosophila (Drosophila suzukii): a global perspective and future priorities, 
J. Pest Sci. (2004). 88 (2015) 469-494. doi:10.1007/s10340-015-0681-z.

[16] O. Rota-Stabelli, M. Blaxter, G. Anfora, Drosophila suzukii, Curr. Biol. 23 (2013) R8-R9. doi:10.1016/j.cub.2012.11.021.

[17] I.W. Keesey, M. Knaden, B.S. Hansson, Olfactory Specialization in Drosophila suzukii Supports an Ecological Shift in Host Preference from Rotten to Fresh Fruit, J. Chem. Ecol. (2015). doi:10.1007/s10886-015-0544-3.

[18] S. Revadi, F. Eccher, V. Mazzoni, S. Al Ani, S. Carlin, U. Vrhovsek, et al., Olfactory responses of Drosophila suzukii to host plant volatiles, Physiol. Entomol. (2015) 117121. doi:10.1111/phen.12088.

[19] D.H. Cha, T. Adams, H. Rogg, P.J. Landolt, Identification and Field Evaluation of Fermentation Volatiles from Wine and Vinegar that Mediate Attraction of Spotted Wing Drosophila, Drosophila suzukii, J. Chem. Ecol. 38 (2012) 1419-1431. doi:10.1007/s10886-012-0196-5.

[20] M.C. Stensmyr, E. Giordano, A. Balloi, A.-M. Angioy, B.S. Hansson, Novel natural ligands for Drosophila olfactory receptor neurones., J. Exp. Biol. 206 (2003) 715-724. doi:10.1242/jeb.00143.

[21] N. Narain, M. de S. Galvão, M.S. Madruga, Volatile compounds captured through purge and trap technique in caja-umbu (Spondias sp.) fruits during maturation, Food Chem. 102 (2007) 726-731. doi:10.1016/j.foodchem.2006.06.003.

[22] M. El Hadi, F.-J. Zhang, F.-F. Wu, C.-H. Zhou, J. Tao, Advances in Fruit Aroma Volatile Research, Molecules. 18 (2013) 8200-8229. doi:10.3390/molecules18078200.

[23] M.C. Stensmyr, T. Dekker, B.S. Hansson, Evolution of the olfactory code in the Drosophila melanogaster subgroup, Proc. R. Soc. London Ser. B-Biological Sci. 270 (2003) 23332340. doi:10.1098/rspb.2003.2512.

[24] T. Dekker, S. Revadi, S. Mansourian, S. Ramasamy, S. Lebreton, P.G. Becher, et al., Loss of Drosophila pheromone reverses its role in sexual communication in Drosophila suzukii, Proc. Biol. Sci. 282 (2015) 20143018. doi:10.1098/rspb.2014.3018.

[25] J. Zhu, K.-C. Park, C. Baker, Thomas, Identification of odors from overripe mango that attract vinegar flies, Drosophila melanogaster, J. Chem. Ecol. 29 (2003).

[26] P. Date, H.K.M. Dweck, M.C. Stensmyr, J. Shann, B.S. Hansson, S.M. Rollmann, Divergence in Olfactory Host Plant Preference in D. mojavensis in Response to Cactus Host Use, PLoS One. 8 (2013) 1-10. doi:10.1371/journal.pone.0070027.

[27] E. Versace, J. Reisenberger, Large-scale assessment of olfactory preferences and learning in Drosophila melanogaster: behavioural and genetic components, PeerJ. 3 (2015) e1214. doi:10.7717/peerj.1214.

[28] M. Lihoreau, I.M. Clarke, J. Buhl, D.J.T. Sumpter, S.J. Simpson, Collective selection of food patches in Drosophila., J. Exp. Biol. 219 (2016) 668-75. doi:10.1242/jeb.127431.

[29] T.F.C. Mackay, S. Richards, E.A. Stone, A. Barbadilla, J.F. Ayroles, D. Zhu, et al., The Drosophila melanogaster Genetic Reference Panel, Nature. 482 (2012) 173-178. doi:10.1038/nature10811.

[30] S.K. Wendakoon, Y. Ueda, Y. Imahori, M. Ishimaru, Effect of short-term anaerobic conditions on the production of volatiles, activity of alcohol acetyltransferase and other quality traits of ripened bananas, J. Sci. Food Agric. 86 (2006) 1475-1480. 
doi:10.1002/jsfa.

[31] A. Antonelli, L. Castellari, C. Zambonelli, A. Carnacini, Yeast influence on volatile composition of wines, J. Agric. Food Chem. 47 (1999) 1139-1144. doi:10.1021/jf9807317.

[32] P.G. Becher, G. Flick, E. Rozpedowska, A. Schmidt, A. Hagman, S. Lebreton, et al., Yeast, not fruit volatiles mediate Drosophila melanogaster attraction, oviposition and development, Funct. Ecol. 26 (2012) 822-828. doi:10.1111/j.1365-2435.2012.02006.x.

[33] S.M.G. Saerens, F.R. Delvaux, K.J. Verstrepen, J.M. Thevelein, Production and biological function of volatile esters in Saccharomyces cerevisiae, Microb. Biotechnol. 3 (2010) 165-177. doi:10.1111/j.1751-7915.2009.00106.x.

[34] P.G. Becher, M. Bengtsson, B.S. Hansson, P. Witzgall, Flying the Fly: Long-range Flight Behavior of Drosophila melanogaster to Attractive Odors, J. Chem. Ecol. 36 (2010) 599607. doi:10.1007/s10886-010-9794-2.

[35] M.R.E. Symonds, B. Wertheim, The mode of evolution of aggregation pheromones in Drosophila species, J. Evol. Biol. 18 (2005) 1253-1263. doi:10.1111/j.14209101.2005.00971.x.

[36] J. Stökl, A. Strutz, A. Dafni, A. Svatos, J. Doubsky, M. Knaden, et al., A deceptive pollination system targeting drosophilids through olfactory mimicry of yeast, Curr. Biol. 20 (2010) 1846-1852. doi:10.1016/j.cub.2010.09.033.

[37] L. Palanca, A.C. Gaskett, C.S. Günther, R.D. Newcomb, M.R. Goddard, Quantifying Variation in the Ability of Yeasts to Attract Drosophila melanogaster, PLoS One. 8 (2013) e75332. doi:10.1371/journal.pone.0075332.

[38] T.A. Markow, The secret lives of Drosophila flies, Elife. 4 (2015) 1-9. doi:10.7554/eLife.06793.

[39] W. Atkinson, B. Shorrocks, Breeding Site Specificity in the Domestic Species of Drosophila, Oecologia. 29 (1977) 223-232. doi:10.1007/BF00345697.

[40] L. Nunney, The colonization of oranges by the cosmopolitan Drosophila, Oecologia. 108 (1996) 552-561.

[41] L. Nunney, Drosophila on Oranges: Colonization, Competition, and Coexistence, Ecology. 71 (1990) 1904-1915.

[42] H.L. Carson, H.D. Stalker, Natural breeding sites for some wild species of Drosophila in the Eastern United States, Ecology. 32 (1951) 317-330. papers2://publication/uuid/91781921-AD21-485D-A4E3-88E7F75B8219.

[43] T. Dobzhansky, M.L. Queal, Genetics of natural populations. I. Chromosome variation in populations of Drosophila pseudoobscura inhabiting isolated mountain ranges, Genetics. 23 (1938) 239-251.

[44] M. Begon, The Relationships of Drosophila obscura Fallén and D. subobscura Collin to Naturally-Occurring fruits, Oecologia. 20 (1975) 255-277. doi:10.1007/BF00347477.

[45] B. Shorrocks, The distribution and abundance of woodland species of British Drosophila (Diptera: Drosophilidae), J. Anim. Ecol. 44 (1975) 851-864. doi:10.2307/3723.

[46] J.C. Lee, D.J. Bruck, H. Curry, D. Edwards, D.R. Haviland, R. a. Van Steenwyk, et al., The susceptibility of small fruits and cherries to the spotted-wing drosophila, Drosophila 
suzukii, Pest Manag. Sci. 67 (2011) 1358-1367. doi:10.1002/ps.2225.

[47] C. Krause Pham, A. Ray, Conservation of Olfactory Avoidance in Drosophila Species and Identification of Repellents for Drosophila suzukii, Sci. Rep. 5 (2015) 11527. doi:10.1038/srep11527.

[48] X.J. Gao, T.R. Clandinin, L. Luo, Sufficient to Mediate Innate Aversion in Drosophila, PLoS One. (2015) 1-14. doi:10.7910/DVN/29507.

[49] M. de Bruyne, K. Foster, J.R. Carlson, Odor Coding in the Antenna, Neuron. 30 (2001) 537-552.

[50] A. Borst, M. Heisenberg, Osmotropotaxis in Drosophila melanogaster, J. Comp. Physiol. 147 (1982) 479-484.

[51] A.-S. Philippe, R. Jeanson, C. Pasquaretta, F. Rebaudo, C. Sueur, F. Mery, Genetic variation in aggregation behaviour and interacting phenotypes in Drosophila, Proc. R. Soc. B Biol. Sci. 283 (2016) 20152967. doi:10.1098/rspb.2015.2967.

[52] M. Lihoreau, I.M. Clarke, J. Buhl, D.J.T. Sumpter, J. Stephen, Collective selection of food patches in Drosophila, J. Exp. Biol. 219 (2016) 668-675.

[53] S. Revadi, S. Lebreton, P. Witzgall, G. Anfora, T. Dekker, P. Becher, Sexual Behavior of Drosophila suzukii, Insects. 6 (2015) 183-196. doi:10.3390/insects6010183.

[54] M. van der Voet, B. Nijhof, M. a. W. Oortveld, A. Schenck, Drosophila models of early onset cognitive disorders and their clinical applications, Neurosci. Biobehav. Rev. 46 (2014) 326-342. doi:10.1016/j.neubiorev.2014.01.013.

[55] E. Versace, G. Vallortigara, Origins of knowledge: Insights from precocial species, Front. Behav. Neurosci. 9 (2015) 338. doi:10.3389/fnbeh.2015.00338.

[56] A. Iacovone, P. Girod, N. Ris, C. Weydert, P. Gibert, M. Poirié, et al., Worldwide invasion by Drosophila suzukii: does being the "cousin" of a model organism really help setting up biological control? Hopes, disenchantments and new perspectives, Rev. d'Ecologie "La Terre La Vie." 70 (2015) 207-214. 
SUPPLEMENTARY MATERIAL

\begin{tabular}{|c|c|c|c|c|c|}
\hline \multirow{2}{*}{$\begin{array}{l}\text { Odor vs. Blank } \\
\text { stimuli }\end{array}$} & \multicolumn{5}{|c|}{ Dose $[\mu \mathrm{g}]$} \\
\hline & 0.002 & 0.02 & 0.2 & 1 & 2 \\
\hline & 22 & 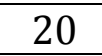 & 28 & 38 & 21 \\
\hline & & & 30 & 42 & 30 \\
\hline E3HB-BLANK & 20 & 30 & 30 & 20 & 20 \\
\hline
\end{tabular}

Supplementary Table 1.

Number of trials per stimulus and dose in the Odor vs. Blank experiment.

\begin{tabular}{|l|c|c|c|}
\hline \multirow{2}{*}{ Odor $\boldsymbol{v} \boldsymbol{s}$. Odor stimuli } & \multicolumn{3}{|c|}{ Dose [ug] } \\
\cline { 2 - 4 } & $\mathbf{0 . 2}$ & $\mathbf{1}$ & $\mathbf{2}$ \\
\hline IAA-BCC & 24 & 20 & 39 \\
\hline E3HB-BCC & 20 & 43 & 39 \\
\hline E3HB-IAA & 21 & 22 & 31 \\
\hline
\end{tabular}

Supplementary Table 2 .

Number of trials by stimuli used and dose in the Odor vs. Odor experiment.

\begin{tabular}{|c|c|c|c|c|c|}
\hline \multirow{2}{*}{$\begin{array}{l}\text { Same odors } \\
\text { stimuli }\end{array}$} & \multicolumn{5}{|c|}{ Dose $[\mu \mathrm{g}]$} \\
\hline & 0.002 & 0.02 & 0.2 & 1 & 2 \\
\hline BCC-BCC & 20 & 20 & 20 & 20 & 20 \\
\hline IAA-IAA & 20 & 20 & 20 & 20 & 20 \\
\hline E3HB-E3HB & 20 & 20 & 20 & 20 & 20 \\
\hline
\end{tabular}

Supplementary Table 3.

Number of trials by stimuli used and dose in the Same odors experiment.

\begin{tabular}{|l|c|c|c|}
\hline \multirow{2}{*}{$\begin{array}{l}\text { Two odors } \boldsymbol{v s .} \text { Blank } \\
\text { stimuli }\end{array}$} & \multicolumn{3}{|c|}{ Dose [ug] } \\
\cline { 2 - 4 } & $\mathbf{0 . 2}$ & $\mathbf{1}$ & $\mathbf{2}$ \\
\hline IAA and BCC - BLANK & 20 & 20 & 20 \\
\hline E3HB and BCC - BLANK & 20 & 20 & 20 \\
\hline E3HB and IAA - BLANK & 24 & 20 & 20 \\
\hline
\end{tabular}

Supplementary Table 4.

Number of trials by stimuli used and dose in the Two odors vs. Blank experiment 